\title{
基于改进蜻蜓算法的斗轮取料机多目标优化
}

\author{
原永亮 ${ }^{1,2}$ 郭正刚 ${ }^{2}$ 王 鹏 $^{3}$ 宋学官 $^{2}$ \\ (1. 河南理工大学机械与动力工程学院 焦作 454003; \\ 2. 大连理工大学机械工程学院 大连 116024; \\ 3. 大连华锐重工集团股份有限公司 大连 116013)
}

\begin{abstract}
摘要: 针对斗轮取料机系统能耗高、重量大、制造成本高、设计变量多等特点, 提出一种改进的蜻蜓算法用以求解斗轮取料 机的多目标优化问题。提出的改进蜻蜓算法基于自然现象和物理现象, 采用空气阻力和库仑力混合组成的策略对传统蜻蜓算 法进行改进, 并通过测试函数验证了改进后蜻蜓算法的性能。然后建立考虑斗轮取料机可靠性和振动频率约束的质量与转动 惯量的多目标优化模型, 利用改进后的蜻蜓算法进行多目标求解, 获得斗轮取料机的 Pareto 前沿解集, 选择以质量与转动惯 量合适权重比为例进行优化, 验证开展斗轮取料机多目标优化的有效性。结果表明, 优化得到的阶梯截面布局方案具有更小 的质量和转动惯量值, 同时可以有效避开斗轮取料机系统的共振问题, 可以使斗轮取料机的性能得到有效改善, 为未来的整 机材料-结构-控制多学科一体化协同优化提供基础。
\end{abstract}

关键词: 蜻蜓算法; 混合策略; 斗轮取料机; 多目标优化

中图分类号: TH122

\section{Multi-objective Optimization of Bucket Wheel Reclaimer Based on Improved Dragonfly Algorithm}

\author{
YUAN Yongliang $^{1,2} \quad$ GUO Zhenggang ${ }^{2} \quad$ WANG Peng ${ }^{3} \quad$ SONG Xueguan $^{2}$ \\ (1. School of Mechanical and Power Engineering, Henan Polytechnic University, Jiaozuo 454003; \\ 2. School of Mechanical Engineering, Dalian University of Technology, Dalian 116024; \\ 3. Dalian Huarui Heavy Industry Group Co., Ltd. Dalian 116013)
}

\begin{abstract}
Aiming at the characteristics of high energy consumption, maximum gross weight, high manufacturing cost and multiple design variables of the bucket wheel reclaimer(BWR), an improved dragonfly algorithm is proposed to solve the multi-objective optimization problem of the BWR. A hybrid strategy composed of air resistance and Coulomb force is proposed to improve the traditional dragonfly algorithm(DA) based on the natural and physical phenomena. The performance of the improved DA is verified by the test functions. A multi-objective optimization model considering the mass and rotational inertia of the BWR's reliability and frequency constraints is established. The improved DA is used for multi-objective solution to obtain the Pareto solution set of the BWR, and a suitable weight ratio of mass and rotational inertia is selected as an example for optimization research. The main purpose is to verify the effectiveness of the multi-objective optimization of the BWR. Results show that the optimized structure layout not only has smaller mass and rotational inertia values, but also can effectively avoid the resonance of the BWR. In addition, it can effectively improve the performance of the BWR, and provide the basis for the future integration of material-structure-control multidisciplinary design optimization.
\end{abstract}

Key words: dragonfly algorithm; hybrid strategy; bucket wheel rclaimer; multi-objective optimization

\section{0 前言}

斗轮取料机是一种对散状物料(颗粒度在 0

\footnotetext{
* 国家自然科学基金资助项目(52075068)。20210110 收到初稿, 20210302 收到修改稿
}

$300 \mathrm{~mm})$ 进行搬运的大型装卸设备, 其具有生产效 率高、运行速度稳定、操作和维护简单等优点, 广 泛应用于港口、冶金、火电厂、化工等行业的物料 搬运。斗轮取料机系统构成复杂, 集成了机械、电 气、液压和控制等多个学科和系统, 在设计与分析 阶段，若直接采用实物模型进行性能分析，计算效 
率低, 很难获得斗轮取料机性能变化规律。为此在 对斗轮取料机进行性能分析和优化设计时, 通常根 据其结构特点将其简化为空间刚架结构。近些年来, 国内外学者对斗轮取料机进行了大量研究。 FILATOV $^{[1]}$ 考虑了斗轮取料机工作过程中不同载荷 的作用, 通过建立其参数化模型并进行仿真计算, 得到了斗轮取料机参数的最佳匹配关系。DORN 等 ${ }^{[2]}$ 提出了判别杆系结构优劣的标准, 有效减少了 通过数学编程篮选结果的次数, 并对杆系的拓扑优 化问题进行了研究。RUSIŃSKI 等 ${ }^{[3]}$ 借助商业有限 元软件对斗轮进行了结构优化设计, 获得了最优的 斗轮结构布局, 有效提高了斗轮的使用时间。

SHANG 等 $^{[4]}$ 基于有限元软件开展了整机结构设计 和模态分析研究, 获得了斗轮取料机系统的固有频 率并对结构改进提出了相应的建议。金华英 ${ }^{[5]}$ 针对 斗轮堆取料机前臂架结构进行了优化与设计分析, 改善了前臂架的受力状况, 对前臂架的改进设计具 有一定的参考价值。YUAN 等 ${ }^{[6-8]}$ 对斗轮取料机开 展了结构与控制一体化设计研究, 获得了取料机结 构、控制以及传动部件参数的最佳组合, 有效降低 了斗轮取料机的系统能耗, 并提高了取料性能。 $\mathrm{ZHAO}^{[9]}$ 将斗轮取料机简化为四杆机构, 建立了斗 轮取料机的优化模型, 采用遗传算法得到了最佳参

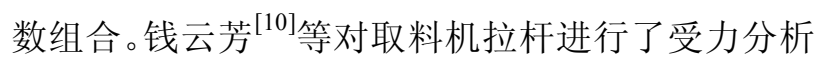
与优化研究, 提出一种交汇式拉杆结构优化方案, 并进行了有限元仿真分析。王九生等 ${ }^{[11]}$ 和吴奋敬 ${ }^{[12]}$ 分别改进了堆取料机回转侧向轨道磨损问题和取 料装置的生产率问题, 对提高斗轮取料机的作业效 率和系统性能具有一定的借鉴意义。LIU 等 ${ }^{[13]}$ 利用 ANSYS 对行走机构开展了静力学分析研究, 并根 据计算结果对腹板进行改进设计, 提高了行走机构 的性能。SUN 等 ${ }^{[14]}$ 采用代理模型技术对斗轮取料 机开展了轻量化研究, 获得了最优结构下的结构参 数组合以及振动规律。龚建民 ${ }^{[15]}$ 和李军 ${ }^{[16]}$ 分别对 斗轮堆取料机开展了总体设计和料斗优化设计研 究, 有效改善了取料机的受力状态。WANG 等 ${ }^{[17]}$ 以最小能耗为优化目标, 对大型矿用挖掘机进行了 结构与控制协同优化研究, 获得最小能耗下结构与 控制参数的最佳组合, 提高了大型矿用挖掘机的综 合性能。

上述研究有效改善了斗轮取料机的关键性能, 为国产斗轮取料机的性能提升做出了重要贡献。然 而斗轮堆取料机性能的进一步优化与提升仍然任重 道远, 主要体现在: 已有斗轮取料机的优化大都是 针对某一特定目标性能, 缺乏对斗轮取料机制造成
本(重量)和使用成本(能耗)的优化研究; 已有研究大 都基于商业软件和传统优化算法, 计算速度和寻优 速度较慢, 无法满足多目标优化需求。因此, 本文 师法自然, 基于自然现象和物理现象提出一种混合 策略对传统蜻蜓算法进行改进, 获得一种可快速寻 优的全局优化算法, 然后对斗轮取料机开展以系统 质量和能耗最小化为目的的多目标优化设计, 同时 将振动频率和结构可靠性考虑在内, 在制造与结构 等的多约束条件下, 进一步提升国产斗轮取料机的 综合性能。

\section{1 混合策略改进的蜻蜓算法}

\section{1 传统的蜻蜓算法}

蜻蜓算法(Dragonfly algorithm, DA)是由澳大利 亚学者 Mirjalilil ${ }^{[18]}$ 提出的, 该算法主要是通过模拟 蜻蜓捕食行为来搜索优化问题全局最优解的, 根据 蜻蜓在捕食过程中的行为, 可以将其分为以下五类， 如图 1 所示。

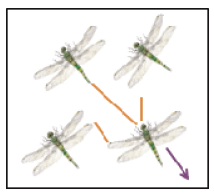

(a) 避撞行为

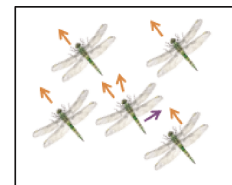

(b) 结队行为

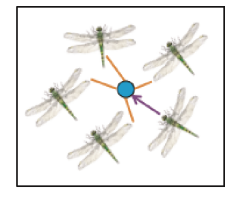

(c) 聚集行为

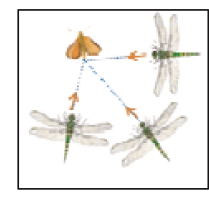

(d) 受食行为

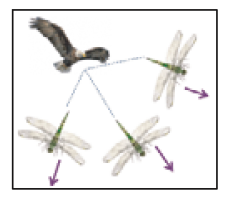

(e) 身朵避行为
图 1 蜻蜓的五种行为

1）避撞行为。蜻蜓在捕食过程中应与其余蜻蜓 保持一定的间隔, 不能发生碰撞, 该行为可以采用 式(1)进行计算

$$
\hat{\boldsymbol{S}}_{i}=-\sum_{j=1}^{N}\left(\boldsymbol{X}-\boldsymbol{X}_{j}\right)
$$

式中, $\hat{\boldsymbol{S}}_{\mathrm{i}}$ 表示第 $i$ 个蜻蜓的分离量, $N$ 表示相邻蜻 蜓的数量, $X$ 和 $\boldsymbol{X}_{j}$ 分别是当前蜻蜓的位置和第 $j$ 个 相邻蜻蜓的位置。

2) 结队行为。为保证全部蜻蜓都能够最快捕食 到目标, 蜻蜓还应与同伴尽可能保持同等速度进行 飞行, 计算如下

$$
\hat{\boldsymbol{A}}_{i}=\frac{\sum_{j=1}^{N} \boldsymbol{V}_{j}}{N}
$$


式中, $\hat{\boldsymbol{A}}_{\mathrm{i}}$ 表示第 $i$ 个蜻蜓的对齐量, $V_{j}$ 表示第 $j$ 个 相邻蜻蜓的速度。

3) 凝聚行为。在捕食过程中应防止某蜻蜓掉 队, 应需尽可能向某个体靠近, 蜻蜓之间还需保持 等间距飞行, 该行为可采用式(3)进行计算

$$
\hat{\boldsymbol{C}}_{i}=\frac{\sum_{j=1}^{N} \boldsymbol{X}_{j}}{N}-\boldsymbol{X}
$$

式中, $\hat{\boldsymbol{C}}_{i}$ 表示第 $i$ 个蜻蜓的凝聚度。

4) 受食行为。一旦某个蜻蜓捕获到目标, 应与 其他蜻蜓分享, 并使其余蜻蜓向其靠拢; 同时该群 体还应在飞行范围内捕获更多食物, 该行为可表 示为:

$$
\hat{\boldsymbol{F}}_{i}=\boldsymbol{X}^{+}-\boldsymbol{X}
$$

式中, $\hat{\boldsymbol{F}}_{i}$ 第 $i$ 个蜻蜓对食物的吸引力, $\boldsymbol{X}^{+}$表示食物 的位置。

5) 躲避行为。蜻蜓群体在捕食过程中应尽可能 避开天敌, 若一旦遇到天敌, 则应该尽快散开飞行, 该行为可以采用式(5)计算

$$
\hat{\boldsymbol{E}}_{i}=\boldsymbol{X}^{-}+\boldsymbol{X}
$$

式中, $\hat{\boldsymbol{E}}_{i}$ 第 $i$ 个蜻蜓逃避天敌的距离, $\boldsymbol{X}^{-}$表示天敌 的位置。

为描述蜻蜓群体在捕食过程中飞行步长和方 向, 引入两个矢量: 步长矢量 $(\Delta \boldsymbol{X})$ 和位置矢量 $\left(\boldsymbol{X}_{\mathrm{t}}\right)$ 。 蜻蜓在飞行捕食过程中的运动的步长和方向, 可用 式(6)计算, 其中 $\Delta \boldsymbol{X}_{\mathrm{t}}$ 表示前一时刻的步长。

$$
\Delta \boldsymbol{X}_{\mathrm{t}+1}=\left(s \hat{\boldsymbol{S}}_{i}+a \hat{\boldsymbol{A}}_{i}+c \hat{\boldsymbol{C}}_{i}+f \hat{\boldsymbol{F}}_{i}+e \hat{\boldsymbol{E}}_{i}\right)+w \Delta X_{t}
$$

式中, $w$ 为惯性权重, $t$ 为迭代计数器, $s$ 表示避撞 分离权重, $a$ 为对齐度权重, $c$ 为凝聚度权重, $f$ 表 示食物的权重, $e$ 是天敌的权重。蜻蜓个体的位置 矢量可采用式(7)进行计算。

$$
\boldsymbol{X}_{t+1}=\boldsymbol{X}_{t}+\Delta \boldsymbol{X}_{\mathrm{t}+1}
$$

为保证蜻蜓在捕食过程中每个蜻蜓都能捕获到 食物, 故需确保每个蜻蜓不是单独飞行。若第 $i$ 个 蜻蜓周围没有同伴时, 该蜻蜓在飞行空间内采用随 机游走方式进行更新步长, 此时蜻蜓可以采用 $L e^{\prime} v y$ 方式飞行，可按照式(8)进行更新。

$$
\boldsymbol{X}_{t+1}=\boldsymbol{X}_{t}+L e^{\prime} v y(d) \times \boldsymbol{X}_{t}
$$

式中, $d$ 是指蜻蜓位置矢量的维数。 $L e^{\prime} v y$ 函数 ${ }^{[19]}$ 可以按照式(9) (10)进行计算

$$
L e^{\prime} v y(x)=0.01 \times \frac{r_{1} \times \sigma}{\left|r_{2}\right|^{\frac{1}{\beta}}}
$$

$$
\beta=\left(\frac{\Gamma(1+\beta) \times \sin \left(\frac{\pi \beta}{2}\right)}{\Gamma(1+\beta) \times \beta \times 2^{\left(\frac{\beta-1}{2}\right)}}\right)^{\frac{1}{\beta}}
$$

式中, $r_{1}$ 和 $r_{2}$ 均为 $[0,1]$ 区间上的随机数, $\Gamma(x)=(x-1)$ !, 且 $\beta$ 是一个常数 ${ }^{[20]}$ 。为清晰表述蜻 蜓算法的寻优流程, 在图 2 中给出蜻蜓算法伪代码。

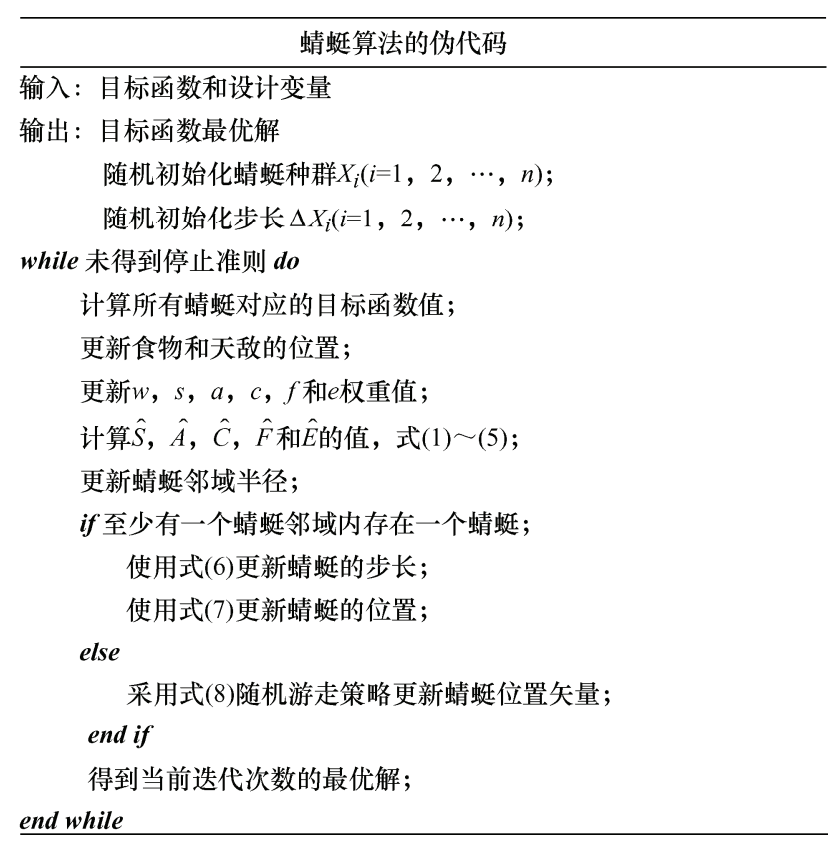

图 2 蜻蜓算法的伪代码

\section{2 混合策略的算法改进}

\subsection{1 基于自然现象的空气阻力策略}

该搜索策略的灵感主要源自大雁在南北迁徙飞 行过程中受到的阻力现象。对应在蜻蜓算法中, 假 设种群 $\boldsymbol{X}_{p}$ 由 $N$ 个蜻蜓组成, 且可以表示为

$$
\boldsymbol{X}_{p}=\left(\boldsymbol{X}_{1}, \cdots, \boldsymbol{X}_{i}, \cdots, \boldsymbol{X}_{N}\right)
$$

蜻蜓在飞行过程中，必然会受到空气阻力的影 响, 根据空气动力学可知, 第 $i$ 个蜻蜓受到的空气 阻力可以表示为

$$
F_{i}=\frac{1}{2} C_{w} \rho_{t} A_{s} V_{i}^{2}
$$

式中, $C_{\mathrm{w}}$ 表示阻力系数, $\rho_{\mathrm{t}}$ 表示蜻蜓所在位置的空 气密度值, $A_{\mathrm{s}}$ 表示受阻面积, $V_{\mathrm{i}}$ 是蜻蜓个体当前的 飞行速度。蜻蜓在飞行过程中, 通常在几米到数百 米高空之间飞行, 飞行高度的差异会引起空气密度 的改变, 该变化可采用式(13)计算 


$$
\rho_{\mathrm{t}}=\rho_{0}\left(\frac{T}{T_{0}}\right)^{4.25588}
$$

式中, $\rho_{0}$ 表示海平面的空气密度值, $T$ 和 $T_{0}$ 分别 表示当前位置和海平面位置的绝对温度。蜻蜓在 飞行过程中会形成尾浴, 该现象会影响后续蜻蜓 飞行过程中的受阻面积, 造成后续的蜻蜓受到的 空气阻力较小。考虑到不同的空气阻力值对寻优 速度具有一定的影响, 为此在空气阻力策略中对 不同蜻蜓的受阻面积也进行了考虑。假设阻力面 积上下界的渐近线值分别为 $A_{l}$ 和 $A_{u}$, 空气阻力 的变化率为 $B, \hat{v}$ 是影响渐近线变化趋势参数, $C_{c}$ 是位于 $(0,1)$ 内的常数, 则不同蜻蜓在飞行寻 优过程中对应的阻力面积可以采用式(14)进行 更新

$$
A_{s}=A_{l}+\frac{A_{u}-A_{1}}{\left(C_{c}+\exp -B\left(x_{i}-x_{1}\right)\right)^{1 / \hat{v}}}
$$

将式(13)和(14)代入式(12), 第 $i$ 个蜻蜓的空气 阻力值可以通过式(15)获得

$$
F_{\mathrm{i}}=\frac{1}{2} C_{w} \rho_{0}\left(\frac{T}{T_{0}}\right)^{4.25588}\left(A_{1}+\frac{A_{\mathrm{u}}-A_{l}}{\left(C_{c}+\exp -B\left(x_{i}-x_{1}\right)\right)^{1 / \mathrm{v}}}\right) V_{\mathrm{i}}^{2}
$$

根据牛顿第二定律, 空气阻力对第 $i$ 个蜻蜓的 影响可以通过计算其加速度获得, 如式(16)所示:

$$
a c_{i}=\frac{F_{i}}{f i t\left(x_{i}\right)}
$$

式中, $\operatorname{fit}\left(x_{i}\right)$ 表示第 $i$ 个蜻蜓在当前迭代中的最优值。

考虑到蜻蜓在飞行过程中受阻面积也是不断变 化的, 因此蜻蜓的凝聚行为系数也随之变化, 其系 数可以按照下式进行更新

$$
c w_{i}=\frac{C_{i}}{C_{1}}
$$

式中, $C_{1}$ 和 $C_{i}$ 分别为凝聚度最初系数和当前迭代系 数的值。结合前文对蜻蜓飞行耐力做出假设可知, 蜻蜓的飞行会随着当前迭代的代数 itear 进行衰减, 如式(18)所示

$$
\mathrm{p}_{\mathrm{i}}=\frac{f i t\left(x_{\mathrm{i}}\right)}{1+\exp -k(\text { iter }-0.5)}
$$

\subsection{2 基于物理现象的库仑力策略}

该搜索策略的灵感主要源自电磁力, 并且假设
蜻蜓之间相互吸引, 而蜻蜓与天敌之间相互排斥。 为保持命名上的一致性，在该搜索策略中每次蜻蜓 迭代获得的适应度值称为蜻蜓的 “质量”。除第一次 迭代外，每个蜻蜓个体的适应度值、整个种群中最 优和最差的适应度的值可以来确定该个体的惯性质 量, 如式(19) (20)所示。

$$
\begin{gathered}
\operatorname{fit}_{i i}(t)=\frac{\text { fit }_{i}(t)-\text { worst }_{i}(t)}{\text { best }_{i}(t)-\text { worst }_{i}(t)} \\
\operatorname{fit}_{i}(t)=\frac{\operatorname{fit}_{i i}(t)}{\sum_{j=1}^{N} f i t_{j}(t)}
\end{gathered}
$$

式中, $f i t_{i}(t)$ 表示第 $i$ 个蜻蜓在第 $t$ 时刻的适应度值, best $_{i}(t)$ 和 worst $_{i}(t)$ 表示第 $t$ 时刻蜻蜓种群内的最优 解和最劣解。

徐遥等 ${ }^{[21]}$ 基于引力算法开展了对每个粒子增 加一个权值的研究, 这样可以使最优个体在下一次 迭代中能够具有较大的惯性, 而惯性质量小的则变 得更小，进而最后得到全局最优解。基于增加权值 的思想, 库仑力搜索策略中也提出一种自适应权值 法，其计算公式如式(21)所示

$\gamma_{i}(t)=\frac{C_{\text {max }}-C_{\text {min }}}{\text { best }_{i}(t)-\text { worst }_{i}(t)}$ fit $_{i}(t)+\frac{C_{\text {min }} \text { best }_{i}(t)-C_{\text {max }} \text { worst }_{i}(t)}{\text { best }_{i}(t)-\text { worst }_{i}(t)}$

式中, $\gamma_{i}(t)$ 是第 $i$ 个蜻蜓的权值, $C_{\text {max }}$ 和 $C_{\text {min }}$ 分别表示 权重的最大系数和最小系数。通过引入权值后, 式 (20)可以修正为式(22)

$$
\operatorname{fit}_{g i}(t)=\operatorname{fit}_{i}\left(t^{\gamma^{\gamma(t}(t)}\right.
$$

蜻蜓个体之间以及蜻蜓与天敌之间的相互作用 力, 如式(23) (24)所示

$$
\begin{gathered}
F_{i j}(t)=\bar{k} \frac{f_{i t_{p i}}(t) \times f i t_{a \mathrm{i}}(t)}{l_{i j}(t)+\varepsilon}\left(x_{j}(t)-x_{i}(t)\right) \\
F_{i e}(t)=-\bar{k} \frac{f_{i t_{p i}}(t) \times \text { enemy }_{i}(t)}{l_{i e}(t)+\varepsilon}\left(x_{e}(t)-x_{i}(t)\right)
\end{gathered}
$$

式中, $f i t_{a i}$ 和 $f i t_{p i}$ 分别表示第 $i$ 个主动蜻蜓和被动蜻 蜓的适应度值, enemy $y_{i}$ 是天敌的适应度值, $\bar{k}$ 是搜 索系数, $l_{i j}$ 表示蜻蜓与蜻蜓之间的距离, $l_{i e}$ 表示蜻 蜓与天敌之间的距离。

为加快库仑力搜索策略的收玫速度, 采用高斯 变异方法, 从而保证收玫速度会随着迭代次数的增 加而变得更快 ${ }^{[22]}$ 。搜索系数可以修正为 


$$
\bar{k}(t)=\bar{k}\left(t_{0}\right) \times \exp (-\hat{\alpha} \times \text { iter } / \text { miter })
$$

式中, $\bar{k}(t)$ 是第 $t$ 时刻的系数, $\bar{k}\left(t_{0}\right)$ 是初始值, $\hat{\alpha}$ 是 指数, 且服从正态分布, iter 和 miter 分别表示当前 迭代次数和最大迭代次数。因此在第 $t$ 时刻的合力 可以用式(26)计算

$$
F_{i}(t)=\sum_{j=1, i \neq j}^{N} \text { rand } \cdot F_{i j}(t)+\sum_{i=1}^{N} \text { rand } \cdot F_{i e}(t)
$$

式中, rand 表示 $[0,1]$ 上的一个随机数。为保证最后 一次迭代时, 只有一个蜻蜓去作用于其他蜻蜓, 本 文引入kbest 参数, 它表示蜻蜓质量按照降序排列的 前 $k$ 个蜻蜓, $k$ 的取值从 $N$ 到 1 随着迭代次数的进 行呈现线性减少, 可采用式(27)进行计算

$$
F_{i}(t)=\sum_{\substack{j \in k b \text { bst } \\ i \neq j}}^{N} \text { rand } \cdot F_{i j}(t)+\sum_{i \in k b e s t}^{N} r a n d \cdot F_{i e}(t)
$$

根据牛顿第二定律, 质量为 $f i t_{g i}(t)$ 的第 $i$ 个蜻蜓 的加速度为

$$
\widehat{a}_{i}(t)=\frac{F_{i}(t)}{f i t_{g i}(t)}
$$

基于空气阻力策略和库仑力策略提出一种混合 策略改进的蜻蜓算法(CF-ARSSDA), 传统蜻蜓算法 中步长计算公式(6)和位置计算公式(7)可以更新为

$$
\boldsymbol{\Delta} \boldsymbol{X}_{t+1}=w \cdot \boldsymbol{\Delta} \boldsymbol{X}_{t}+\left(a c_{i} \hat{\boldsymbol{S}}_{i}+a c_{i} \hat{\boldsymbol{A}}_{i}+c w_{i} \hat{\boldsymbol{C}}_{i}+f \hat{\boldsymbol{F}}_{i}+e \hat{\boldsymbol{E}}_{i}\right) \times p_{i}+\hat{a}_{i}(t)
$$

$$
\boldsymbol{X}_{t+1}=\boldsymbol{X}_{t}+\Delta \boldsymbol{X}_{t+1}
$$

改进后蜻蜓算法的演化过程如下。

步骤 1: 首先, 对蜻蜓搜索算法中的参数进行 初始化, 包括: 蜻蜓的种群数 $N$; 最大的迭代次数 miter; 优化问题中设计变量的维数; 基本惯性参 数 $w$; 邻域半径 $r$ 等。

步骤 2: 采用随机生成矩阵的方式对蜻蜓种群 的初始位置和步长矢量进行初始化。

步骤 3: 开始第一次迭代, 令iter $=1$ 。

步骤 4: 计算所有蜻蜓的适应度函数值, 根据 蜻蜓适应度值确定食物和天敌的位置。

步骤 5: 根据公式计算避撞、结队、聚集、受 食和躲避的五个因素的值。

步骤 6: 计算蜻蜓当前位置的空气密度和受阻 面积, 根据空气阻力公式计算蜻蜓的加速度值。

步骤 7: 计算蜻蜓之间的欧式距离并更新当代 蜻蜓避撞、结队、聚集、受食和躲避的系数和蜻蜓
的飞行耐力系数。

步骤 8: 根据适应度值, 更新最优和最差个体 的值, 并更新“库仑力常数”的值。

步骤 9: 计算蜻蜓的权重值, 并根据权重值计 算蜻蜓的惯量质量。

步骤 10: 运用库仑力和牛顿第二定律公式, 依 次计算出蜻蜓的合力和加速度值。

步骤 11: 更新蜻蜓周围的搜索半径。

步骤 12: 判断蜻蜓个体周围是否至少有一个蜻 蜓存在, 若有蜻蜓存在, 按照式(29)更新步长; 若 周围不存在蜻蜓, 则蜻蜓按照随机游走式(8)进行更 新。

步骤 13: 检查每个蜻蜓是否在可行域范围内飞 行, 若不在可行域范围内, 分别按照可行域的上下 界进行修正, 保证每个蜻蜓都在可行域范围内飞行。

步骤 14: 判断是否满足终止搜索准则, 在满足 终止准则时, 输出最优解并结束; 若不满足终止准 则条件, 则令 iter $=i t e r+1$, 并跳回到步骤 4 。

改进后的蜻蜓算法充分考虑了蜻蜓与蜻蜓、蜻 蜓与天敌之间的相互作用, 有效改善了寻优过程中 线性步长搜索的不足。为便于理解改进后蜻蜓的优 势, 图 3 和图 4 给出了其伪代码和寻优流程图。

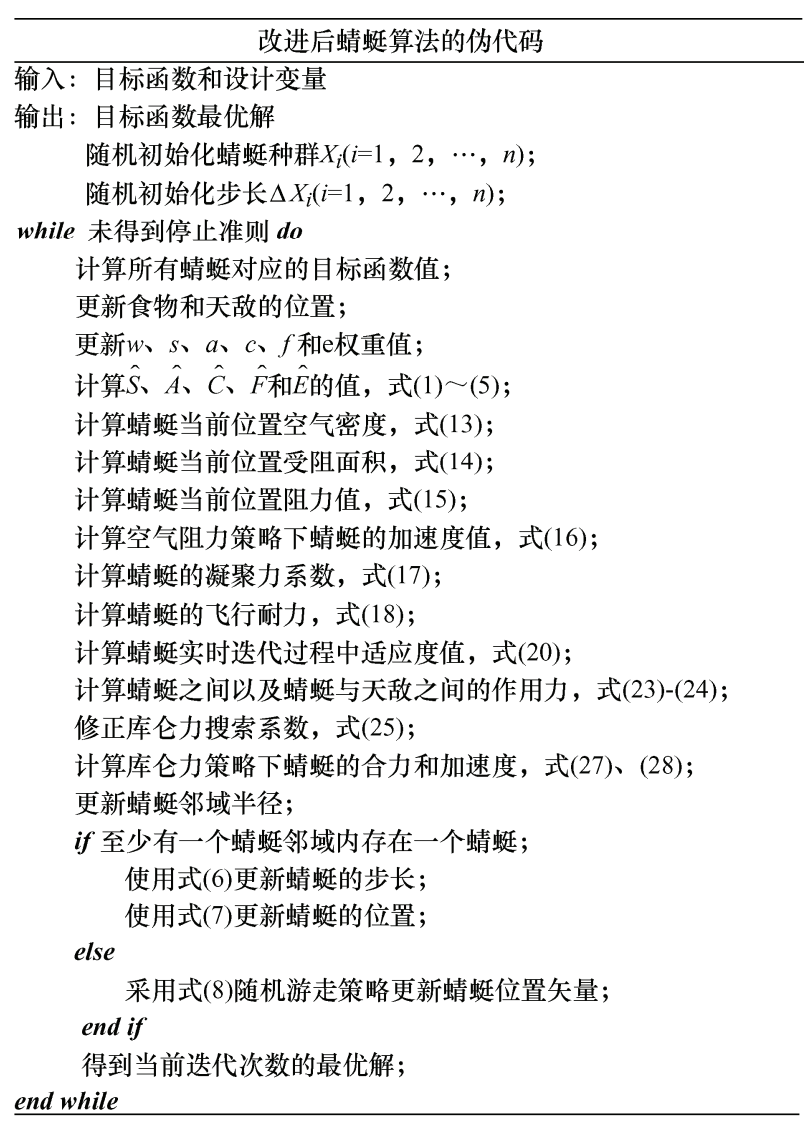

图 3 改进后蜻蜓算法的伪代码 


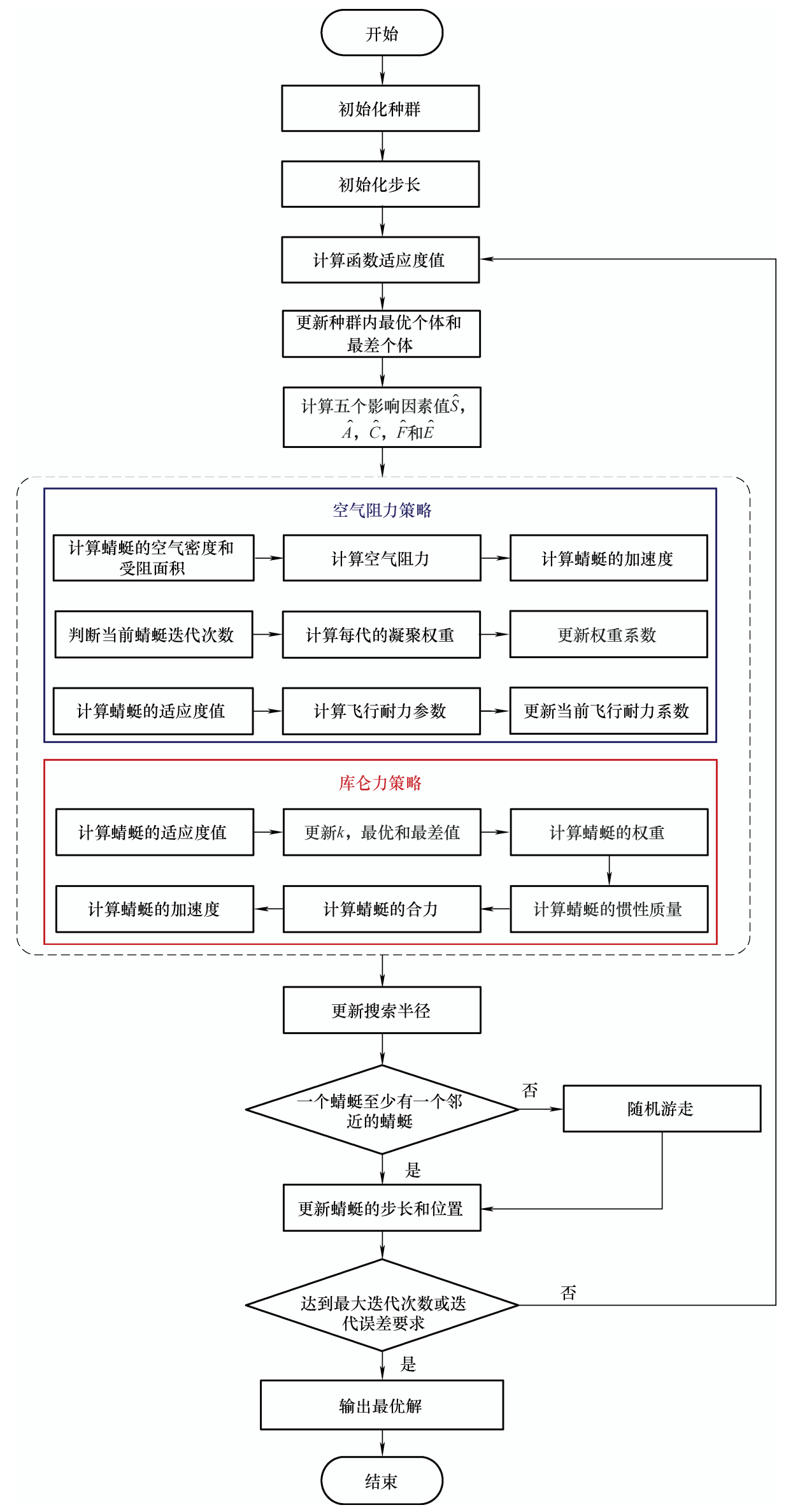

图 4 改进后蜻蜓算法的寻优流程图 


\section{3 算法性能验证}

选取了 6 个常用的基准函数进行算法性能验 证, 测试函数如表 1 所示, 其中包括单峰、多峰、 低维多峰等不同的测试函数。为验证改进后算法的 性能，同时选取先进的飞蛾扑火优化算法 (Moth-flame optimization, MFO) ${ }^{[23-24]}$ 、协方差自适应 调整的进化策略 (Covariance matrix adaptation evolution strategy, CMA-ES) ${ }^{[25]}$ 、精英反向蜻蜓算法 (Elite opposition learning and exponential function steps-based dragonfly algorithm, EOEDA) ${ }^{[26]}$ 进行对 比分析, 其测试函数结果如表 2 所示。

表 1 测试函数

\begin{tabular}{cccc}
\hline 测试函数 & 维数 & 区间 & $f_{\min }$ \\
\hline$f_{1}(x)=\sum_{i=1}^{n} x_{i}^{2}$ & 20 & {$[-100,100]$} & 0 \\
$f_{2}(x)=\sum_{i=1}^{n}\left|x_{i}\right|+\prod_{i=1}^{n}\left|x_{i}\right|$ & 20 & {$[-100,100]$} & 0 \\
$f_{3}(x)=\sum_{i=1}^{n}\left[x_{i}^{2}-10 \cos \left(2 \pi x_{i}\right)+10\right]$ & 20 & {$[-5.12,5.12]$} & 0 \\
$f_{4}(x)=\frac{1}{4000} \sum_{i=1}^{n} x_{i}^{2}-\prod_{i=1}^{n} \cos \left(\frac{x_{i}}{\sqrt{i}}\right)+1$ & 20 & {$[-50,50]$} & 0 \\
$f_{5}(x)=4 x_{1}^{2}-2.1 x_{1}^{4}+\frac{1}{3} x_{1}^{6}+x_{1} x_{2}-4 x_{2}^{2}+4 x_{2}^{4}$ & 2 & {$[-5,5]$} & -1.032 \\
$f_{6}(x)=\left(x_{2}-\frac{5.1}{4 \pi^{2}} x_{1}^{2}+\frac{5}{\pi} x_{1}-6\right)^{2}+10\left(1-\frac{1}{8 \pi}\right) \cos x_{1}+10$ & 2 & {$[-5,5]$} & 0.398 \\
\hline
\end{tabular}

表 2 测试函数结果

\begin{tabular}{|c|c|c|c|c|c|c|}
\hline 函 & & MFO & CMA-ES & DA & EOEDA & $\begin{array}{c}\text { CF-ARSSD } \\
\text { A }\end{array}$ \\
\hline \multirow[b]{2}{*}{$f_{1}$} & 均值 & & $3.126 \times 10^{-20}$ & $1.203 \times 10^{1}$ & $4.245 \times 10^{-14}$ & ${ }^{4} 1.167 \times 10^{-17}$ \\
\hline & 方差 & $1.632 \times 10^{-8}$ & $1.519 \times 10^{-21}$ & $1.490 \times 10^{0}$ & $1.134 \times 10^{-15}$ & $54.661 \times 10^{-18}$ \\
\hline \multirow{2}{*}{$f_{2}$} & 均值 & $6.919 \times 10^{-8}$ & $3.618 \times 10^{-9}$ & $1.331 \times 10^{0}$ & $5.902 \times 10^{-9}$ & $1.095 \times 10^{-8}$ \\
\hline & 方差 & $4.396 \times 10^{-9}$ & $9.491 \times 10^{-10}$ & $6.833 \times 10^{-1}$ & $7.116 \times 10^{-10}$ & ${ }^{0} 2.380 \times 10^{-9}$ \\
\hline \multirow[b]{2}{*}{$f_{3}$} & 均值 & $3.961 \times 10^{-1}$ & 0.000 & $9.441 \times 10^{1}$ & 0 & 0 \\
\hline & 方差 & $2.913 \times 10^{-2}$ & 0.000 & $3.953 \times 10^{0}$ & 0 & 0 \\
\hline \multirow[b]{2}{*}{$f_{4}$} & 均值 & $2.151 \times 10^{-3}$ & 0.000 & $9.811 \times 10^{-1}$ & 0 & 0 \\
\hline & 方差 & $8.910 \times 10^{-4}$ & 0.000 & $1.305 \times 10^{-1}$ & 0 & 0 \\
\hline \multirow[b]{2}{*}{$f_{5}$} & 均值 & $-1.032 \times 10^{0}$ & $-1.032 \times 10^{0}$ & $-1.032 \times 10^{0}$ & $-1.032 \times 10^{0}$ & ${ }^{0}-1.032 \times 10^{0}$ \\
\hline & 方差 & $-5.614 \times 10^{-4}$ & ${ }^{4} 3.014 \times 10^{-5}$ & $4.621 \times 10^{-4}$ & $2.017 \times 10^{-5}$ & $3.743 \times 10^{-5}$ \\
\hline & 均值 & $3.980 \times 10^{-1}$ & $3.980 \times 10^{-1}$ & $3.980 \times 10^{-1}$ & $3.980 \times 10^{-1}$ & $3.980 \times 10^{-1}$ \\
\hline & 方差 & $5.079 \times 10^{-2}$ & $1.097 \times 10^{-2}$ & $4.273 \times 10^{-6}$ & $7.004 \times 10^{-7}$ & $3.394 \times 10^{-14}$ \\
\hline
\end{tabular}

通过对比表 2 中不同优化算法的结果可知, CMA-ES 算法在 $f_{1}$ 测试函数获得的最优解要明显优 于其余优化算法，而在其余的测试函数中, CF-ARSSDA 的性能均明显优于其他优化算法。为 对比不同优化算法的性能, 选取了 $f_{1} 、 f_{2} 、 f_{5}$ 和 $f_{6}$ 四
个进行对比, 图 5 和图 6 给出了五种优化算法的迭 代曲线图和计算时间。

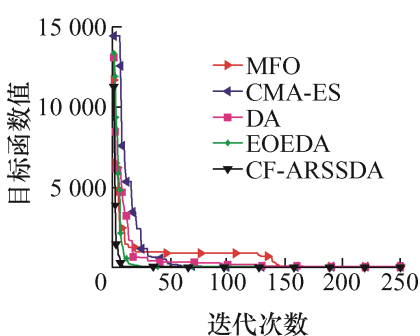

(a) $f_{1}$

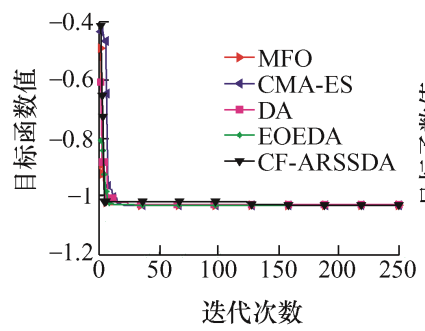

(c) $f_{5}$

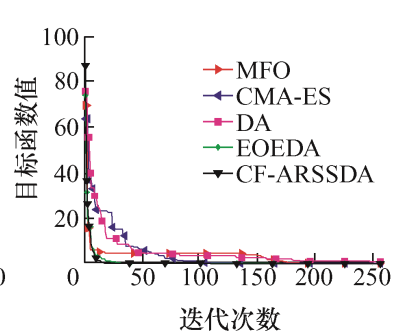

(b) $f_{2}$

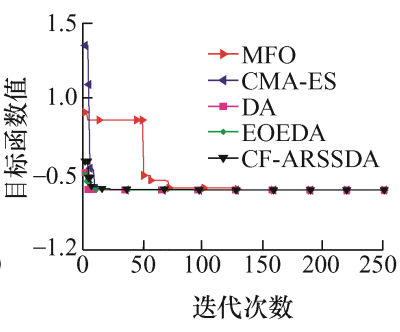

(d) $f_{6}$
图 5 不同优化算法的迭代图

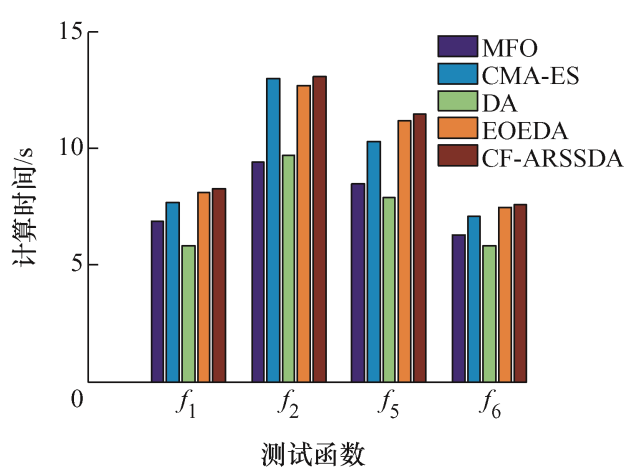

图 6 不同优化算法的计算时间

从图 5 和图 6 可知, CF-ARSSDA 在收玫速度 上有明显优于其他算法, 主要原因是 CF-ARSSDA 算法使用了先进的搜索策略，同时考虑蜻蜓本身以 及蜻蜓之间的相互作用关系; 值得注意的是, CF-ARSSDA 算法与其他算法计算相同迭代次数的 总体计算耗时也相对较长。综合上述测试函数集可 得, CF-ARSSDA 的收玫性和鲁棒性要优于其他策 略改进的蜻蜓算法, 因此 CF-ARSSDA 可以作为一 种高效的优化算法求解工程优化问题。

\section{2 斗轮取料机多目标优化建模}

\section{1 目标函数及设计变量的确定}

斗轮取料机结构复杂、跨度大, 由多段焊接 而成, 但是总体上, 可以将其简化为典型的刚架 结构, 如图 7 所示。考虑到斗轮取料机的焊接工 
艺和结构优化的复杂程度, 对于不同位置相同板 件尺寸可以选取一个设计变量。本文根据组成斗 轮取料机杆件的尺寸和结构参数类型进行分类, 可以将杆件的面积分为 8 类 $\left(A_{s 1}, A_{s 2}, \cdots, A_{s 8}\right)$, 可 变杆件的长度分为 9 类 $\left(L_{1}, L_{2}, \cdots, L_{9}\right)$, 不同支撑 位置梁的宽和高 $\left(H_{i}, W_{i}, i=1,2,3\right)$; 同时, 还存在 影响结构布局的 $\left(\alpha_{1}, \alpha_{2}, \cdots, \alpha_{4}\right) 4$ 个角度参数。每 种工字梁面积有 4 个参数, 不同拉杆的面积有 2 个参数。最终, 影响取料机结构参数变量共计 45 个变量。

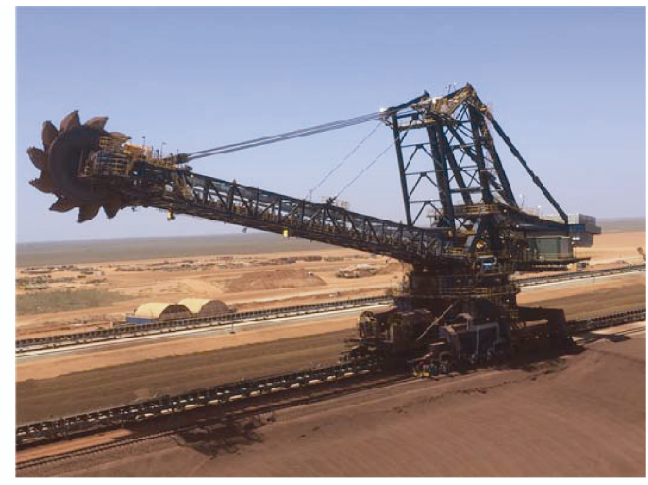

(a) 斗轮取料机实物图

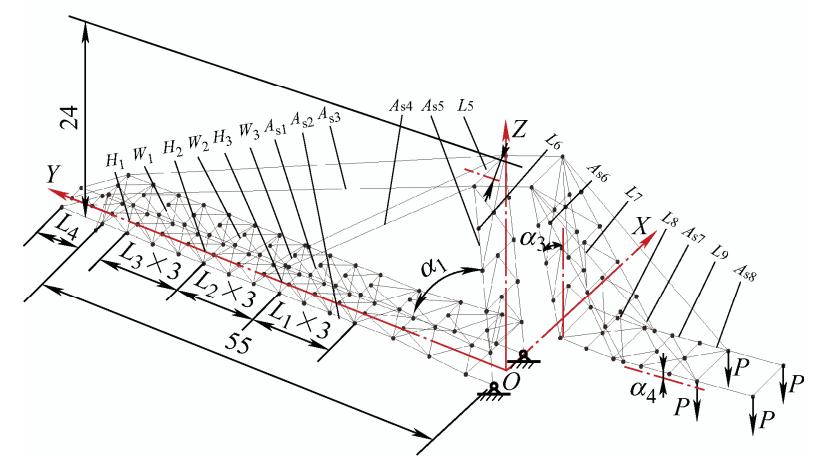

(b) 斗轮取料机简化图 (m)

图 7 斗轮取料机结构示意图

以往斗轮取料机的优化设计研究集中在尺寸、 形貌等单目标优化方面, 并未考虑使用过程中的巨 大转动惯量和质量因素的影响。斗轮取料的取料作 业主要依靠悬臂的旋转运动来实现, 而转动惯量是 影响工作过程中能耗大小的主要因素, 质量则是影 响系统造价甚至振动的关键因素。因此, 斗轮取料 机的多目标优化模型中的目标函数可以分别选取为 系统的总质量和转动惯量。其多目标优化数学模型 的目标函数可以表示为

$$
\left\{\begin{array}{l}
\min M_{\text {total }}=\sum_{i=1}^{\bar{n}} \rho \cdot A_{i} \cdot l_{i} \\
\min J_{\text {total }}=\sum_{i=1}^{\bar{n}} J_{c i}+\rho \cdot A_{i} \cdot l_{i} \cdot \bar{r}_{i}
\end{array}\right.
$$

式中, $\bar{n}$ 表示杆件的数量, $\rho$ 表示杆件的材料密度, $A_{i}$ 表示成第 $i$ 个杆件的面积, $l_{i}$ 表示第 $i$ 个杆件的长 度, $\bar{r}_{i}$ 表示第 $i$ 个杆件距离回转中心的距离, $J_{c i}$ 表 示通过第 $i$ 个杆件质心转轴的转动惯量。

\section{2 约束条件的确定}

斗轮取料机的优化是多目标问题, 同时也是典 型的多约束和强约束问题, 除了需要对斗轮取料 机的基本尺寸进行约束来保证满足工作场合的要 求, 还需要对部件的可靠性进行约束, 这是保证 系统长期稳定运行的关键要素。除此之外, 根据 用户的设计要求，优化后的结构的固有频率还需 要避开实际作业时的工作频率。综上所述, 斗轮 取料机多目标优化的约束条件包括: 设计变量取 值范围、悬臂总长、杆件位移可靠性约束、杆件 应力可靠性约束以及以斗轮取料机固有频率约 束, 其约束条件如下:

$$
\left\{\begin{array}{l}
x_{\text {min }} \leqslant x \leqslant x_{\text {max }} \\
p_{\delta^{*}}-p\left(\delta \leqslant a_{*}\right) \leqslant 0 \\
p_{\sigma^{*}}-p\left(\sigma \leqslant\left[\sigma_{*}\right]\right) \leqslant 0 \\
\bar{f}_{q} \geqslant \bar{f}_{q}^{\min } \\
\bar{f}_{r} \leqslant \bar{f}_{r}^{\max } \\
R=R_{q}
\end{array}\right.
$$

式中, $p_{\delta^{*}}$ 和 $p_{\sigma^{*}}$ 分别表示满足最低要求的杆件位移 可靠性概率和应力可靠性概率, $a_{*}$ 是斗轮取料机杆 件的最大位移量, $[\sigma *$ 表示材料允许的最大应力值, $\bar{f}_{q}$ 和 $\bar{f}_{q}^{\text {min }}$ 分别表示第 $q$ 阶固有频率和最小要求频 率, $\bar{f}_{r}$ 和 $\bar{f}_{r}^{\text {min }}$ 分别表示第 $r$ 阶固有频率和最大要求 频率, $R$ 和 $R_{q}$ 分别表示斗轮取料机悬臂优化前和优 化后的长度。

在给定位移界限值前提下, 斗轮取料机的可靠 性约束方程可以表示为

$$
\mathrm{p}_{\delta^{*}}-p\left(\delta \leqslant a_{*}\right) \leqslant 0
$$

由于节点的位移量 $\delta$ 服从正态分布, 则 $\frac{\delta-\mu_{s}}{\sigma_{\delta}}$ 服从标准正态分布，结合正态分布性质可得

$$
\begin{gathered}
p\left(\sigma \leqslant S_{*}\right)=\Phi\left(\frac{\mathrm{a}-\mu_{\delta}}{\sigma_{\delta}}\right) \\
\beta_{\delta}=\Phi^{-1}\left(p_{\delta^{*}}\right)
\end{gathered}
$$

式中, $\mu_{\delta}$ 和 $\sigma_{\delta}$ 分别表示期望值和标准差, $\Phi^{-1}$ 表示 正态分布的反函数， $\beta_{\delta}$ 表示系统位移的可靠度指 标。系统位移可靠性要求可以将原来可靠性约束 转化为 


$$
\frac{a_{*}-\mu_{\delta}}{\sigma_{\delta}} \geqslant \beta_{\delta}
$$

将上式的约束转化为位移可靠性约束下对应的 可靠性系数 $k_{\delta}$, 可以表示为

$$
k_{\delta}=1+\frac{\sigma_{\delta}}{\mu_{\delta}} \beta_{\delta}
$$

通过对可靠性约束进行变形, 斗轮取料机工作 装置原可靠性约束可以采用式(38)进行描述

$$
k_{\delta} \cdot \mu_{\delta}-a_{*} \leqslant 0
$$

应力可靠性约束处理方法与位移可靠性约束处 理的方法类似, 根据正态分布函数的性质以及应力强度干涉理论可得式(39)和(40)

$$
\begin{aligned}
p\left(\sigma \leqslant S_{*}\right) & =\Phi\left(\frac{\mu_{S}-\mu_{\sigma}}{\sqrt{\sigma_{S}^{2}+\sigma_{\sigma}^{2}}}\right) \\
\beta_{\sigma} & =\Phi^{-1}\left(p_{\sigma^{*}}\right)
\end{aligned}
$$

式中, $S_{*}$ 表示强度服从正态分布, $\mu_{s}$ 和 $\sigma_{s}$ 分布表 示强度的期望值和标准差, $\mu_{\sigma}$ 和 $\sigma_{\sigma}$ 分别表示应力 的期望值和标准差。斗轮取料机系统应力的可靠性 约束可以将其进行转化, 如式(41)所示

$$
\beta_{\sigma} \cdot \sqrt{\sigma_{S}^{2}+\sigma_{\sigma}^{2}}-\mu_{S}+\mu_{\sigma} \leqslant 0
$$

采用处理位移约束的方法, 并引入应力和强度 的变异系数 $v_{\sigma}$ 和 $v_{S}$, 定义杆件应力可靠性安全系数 为 $k_{\sigma}=\mu_{s} / \mu_{\sigma}$, 则式(41)可以改写为

$$
\beta_{\sigma} \cdot \sqrt{\mathrm{k}_{\sigma}^{2} \cdot v_{S}^{2}+v_{\sigma}^{2}}-k_{\sigma}+1 \leqslant 0
$$

式(42)的临界方程可以看做是 $k_{\sigma}$ 的一元二次方 程, 其解为

$$
k_{\sigma}=\frac{1+\beta_{\sigma} \sqrt{v_{S}^{2}+v_{\sigma}^{2}-\beta_{\sigma} \cdot v_{S}^{2} \cdot v_{\sigma}^{2}}}{1-\beta_{\sigma}^{2} \cdot v_{S}^{2}}
$$

由式(43)可知, 这与传统力学上的结构安全系 数设计已不相同, 可靠性安全系数是由可靠度指标、 应力变异系数和强度变异系数共同决定的。系统的 原可靠性约束可以近似采用式(44)表示

$$
k_{\sigma} \cdot \mu_{\sigma}-\mu_{S} \leqslant 0
$$

对于斗轮取料机有阻尼结构系统, 其运动方程 可以表述为

$$
\boldsymbol{M} \ddot{\boldsymbol{\delta}}+\boldsymbol{C} \dot{\boldsymbol{\delta}}+\boldsymbol{K} \boldsymbol{\delta}=\boldsymbol{F}
$$

式中, $M 、 K 、 C$ 分别表示系统的质量矩阵、刚度矩 阵以及阻尼矩阵, $\boldsymbol{F}$ 为系统节点载荷矢量, $\ddot{\boldsymbol{\delta}} 、 \dot{\boldsymbol{\delta}} 、 \boldsymbol{\delta}$ 分别为系统中加速度矢量、速度矢量以及位移矢量。 根据式(45)可知, 在外力为零时, 取料机系统从初始
状态到开始振动过程中的微分方程可以表示为 ${ }^{[27]}$

$$
\boldsymbol{M} \ddot{\delta}+\boldsymbol{C} \dot{\delta}+\boldsymbol{K} \delta=\mathbf{0}
$$

若将系统中阻力忽略, 则式(46)的特解为 ${ }^{[27]}$

$$
\delta=\delta_{r} \sin (\omega t+\theta)
$$

式中, $\delta r$ 为特征矢量, 将式(47)代入至阻尼为零的 微分方程中可以得到

$$
\left(\boldsymbol{K}-\bar{f}^{2} \boldsymbol{M}\right) \delta=\boldsymbol{0}
$$

由于斗轮取料机存在非零解, 工作装置的特征 矩阵可表示为 $\breve{\boldsymbol{B}}=\boldsymbol{K}-\bar{f}^{2} \boldsymbol{M}$ 。根据线性代数相关理 论可知式(48)为齐次线性方程组, 且有:

$$
\breve{\boldsymbol{B}}=\left(\begin{array}{cccc}
K_{11}-\bar{f}^{2} M_{11} & K_{12}-\bar{f}^{2} M_{12} & \cdots & K_{1 n}-\bar{f}^{2} M_{1 n} \\
K_{21}-\bar{f}^{2} M_{21} & K_{22}-\bar{f}^{2} M_{22} & \cdots & K_{2 n}-\bar{f}^{2} M_{2 n} \\
\vdots & \vdots & \vdots & \vdots \\
K_{n 1}-\bar{f}^{2} M_{n 1} & K_{n 2}-\bar{f}^{2} M_{n 2} & \cdots & K_{n n}-\bar{f}^{2} M_{n n}
\end{array}\right)=\boldsymbol{0}
$$

化简工作装置特征矩阵行列式为零的方程, 可 得斗轮取料机的特征方程:

$$
\bar{f}^{2 n}+a_{1} \bar{f}^{2 n-2}+\cdots+a_{n-1} \bar{f}^{2}+a_{n}=0
$$

求解方程可以得到第 $i$ 阶固有振动频率 $\bar{f}_{i}$, 然 后将各阶的固有频率带入到斗轮取料机的系统方 程, 得到各阶特征方程的解和对应的特征矢量。由 此可见, 求解固有振动频率和振型实际上是计算系 统方程的特征值和特征矢量。对于斗轮取料机的优 化问题, 频率约束可以转化为

$$
\begin{aligned}
& \bar{f}_{q}^{\text {min }} / \bar{f}_{q}-1 \leqslant 0 \\
& \bar{f}_{r} / \bar{f}_{r}^{\text {max }}-1 \leqslant 0
\end{aligned}
$$

最后, 斗轮取料机的约束可以描述为

$$
\left\{\begin{array}{l}
x_{\min } \leqslant x \leqslant x_{\text {max }} \\
k_{\delta} \cdot \mu_{\delta}-a \leqslant 0 \\
k_{\sigma} \cdot \mu_{\sigma}-\mu_{S} \leqslant 0 \\
\bar{f}_{q}^{\min } / \bar{f}_{q}-1 \leqslant 0 \\
\bar{f}_{r} / \bar{f}_{r}^{\max }-1 \leqslant 0 \\
R=R_{q}
\end{array}\right.
$$

\section{3 多目标优化结果与性能评估}

求解多目标优化问题的方法较多，本节将多目 标优化问题转化为 Pareto 最优解问题 ${ }^{[28]}$ 。简单来说, Pareto 最优解问题是采用优化算法生成大量的非支 
配解, 构成非支配解集, 决策者可以根据实际情况 从非支配解集中得到所需的最优解, Pareto 最优解 可以理解为设计变量集里面不存在某设计变量可以 支配另一设计变量 ${ }^{[29]}$ 。斗轮取料机的 Pareto 前沿如 图 8 所示。

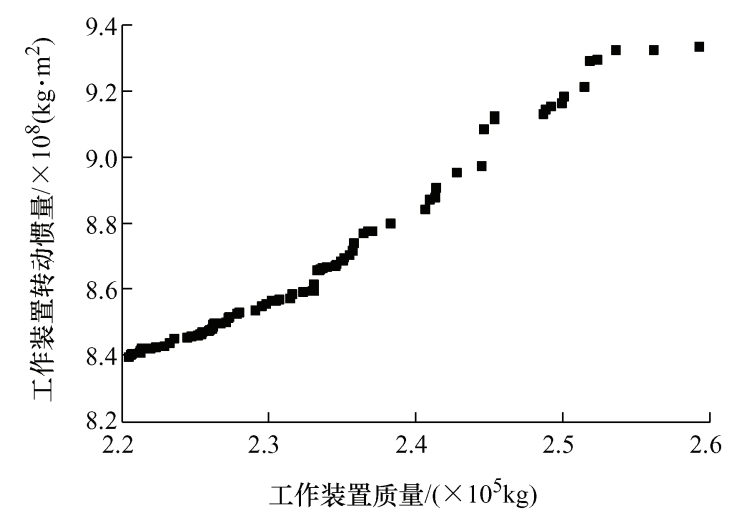

图 8 Pareto 解集曲线图

从图 8 可知, 斗轮取料机经过迭代后形成了一 条可以支配值域的 Pareto 解集曲线, 图中的每个 点都表示满足要求的一组最优解。由图中数据明 显可知, 斗轮取料机的质量和转动惯量并不是线 性变化关系, 主要是因为斗轮取料机尾部配有配 重块, 而结构尺寸变化对转动惯量的影响程度要 大于对系统质量的影响程度, 反之亦然。由于取 料机系统质量增加引起的成本要远小于工作过程 中巨大转动惯量引起电能消耗以及电机损耗成 本, 因此在以后的改进设计中, 应优先考虑转动 惯量的影响。

Pareto 解集为取料机的优化提供了多样性选 择, 但是最终产品通常要根据最终目的或者用户与 设计人员的经验选择其中一个解。根据对已有斗轮 取料机的数据统计, 若按 $8 \mathrm{~h} /$ 天, 250 300 天/年连续 工作 10 年计算, 斗轮取料机工作装置的质量 (材料) 成本远低于转动所需要的能耗成本, 甚至不到 $1 / 4$ 。 因此, 本文将质量与转动惯量的权重比定为 $2: 8$ 进 行最终优化, 优化的结果如表 3 所示。

图 9 为斗轮取料机优化前和优化后结构布局 的变化。显然可知, 优化后斗轮取料机悬臂结构为 阶梯截面结构。对比图 $9 \mathrm{a}$ 和 $9 \mathrm{~b}$ 中的数据, 优化前 和优化后斗轮取料机悬臂的长度保持不变, 仍为初 始结构长度 $55 \mathrm{~m}$, 这主要是为了保证斗轮取料机 优化后依然满足取料场地要求; 平衡架的长度由 $33.470 \mathrm{~m}$ 缩短至 $27.014 \mathrm{~m}$, 降低了 $19.289 \%$; 同时, 斗轮取料高度也由初始 $23.550 \mathrm{~m}$ 降低至 $21.981 \mathrm{~m}$, 降低了 $6.662 \%$ 。
表 3 优化结果

\begin{tabular}{|c|c|c|c|}
\hline 设计变量 & 初始参数 & 优化结果 & 变化幅度 $(\%)$ \\
\hline$L_{1} / \mathrm{m}$ & 3.000 & 2.862 & +4.617 \\
\hline$L_{2} / \mathrm{m}$ & 3.000 & 3.044 & +0.133 \\
\hline$L_{3} / \mathrm{m}$ & 3.000 & 2.504 & -16.533 \\
\hline$L_{4} / \mathrm{m}$ & 3.900 & 4.565 & +17.038 \\
\hline$L_{5} / \mathrm{m}$ & 6.800 & 7.204 & +5.934 \\
\hline$L_{6} / \mathrm{m}$ & 4.800 & 5.069 & +5.594 \\
\hline$L_{7} / \mathrm{m}$ & 4.370 & 3.927 & -10.149 \\
\hline$L_{8} / \mathrm{m}$ & 3.700 & 3.428 & -7.351 \\
\hline$L_{9} / \mathrm{m}$ & 3.770 & 3.054 & -19.005 \\
\hline$A_{\mathrm{s} 1} / \mathrm{m}^{2}$ & $1.080 \times 10^{-2}$ & $1.142 \mathrm{e} \times 10^{-2}$ & +5.694 \\
\hline$A_{\mathrm{s} 2} / \mathrm{m}^{2}$ & $3.500 \times 10^{-2}$ & $4.117 \times 10^{-2}$ & +17.614 \\
\hline$A_{\mathrm{s} 3} / \mathrm{m}^{2}$ & $5.000 \times 10^{-3}$ & $4.485 \times 10^{-3}$ & -10.310 \\
\hline$A_{\mathrm{s} 4} / \mathrm{m}^{2}$ & $5.350 \times 10^{-2}$ & $5.164 \times 10^{-2}$ & -3.486 \\
\hline$A_{\mathrm{s} 5} / \mathrm{m}^{2}$ & $1.080 \times 10^{-2}$ & $1.214 \times 10^{-2}$ & +12.361 \\
\hline$A_{\mathrm{s} 6} / \mathrm{m}^{2}$ & $1.080 \times 10^{-2}$ & $1.111 \times 10^{-2}$ & +2.778 \\
\hline$A_{\mathrm{s} 7} / \mathrm{m}^{2}$ & $4.290 \times 10^{-3}$ & $4.737 \times 10^{-3}$ & +10.048 \\
\hline$A_{\mathrm{s} 8} / \mathrm{m}^{2}$ & $8.500 \times 10^{-3}$ & $7.053 \times 10^{-3}$ & -17.029 \\
\hline$H_{1} / \mathrm{m}$ & 3.500 & 2.863 & -18.200 \\
\hline$H_{2} / \mathrm{m}$ & 3.500 & 3.085 & -11.871 \\
\hline$H_{3} / \mathrm{m}$ & 3.500 & 4.195 & +19.857 \\
\hline$W_{1} / \mathrm{m}$ & 3.00 & 2.805 & -6.500 \\
\hline$W_{2} / \mathrm{m}$ & 3.000 & 2.817 & -6.117 \\
\hline$W_{3} / \mathrm{m}$ & 3.000 & 3.257 & +8.567 \\
\hline$\alpha_{1 /}\left({ }^{\circ}\right)$ & 70.000 & 69.400 & -0.858 \\
\hline$\alpha_{2 /}\left(^{\circ}\right)$ & 7.780 & 7.751 & -0.379 \\
\hline$\alpha_{3 /}\left({ }^{\circ}\right)$ & 26.000 & 20.424 & -21.487 \\
\hline$\alpha_{4} /\left(^{\circ}\right)$ & 7.200 & 7.230 & +0.417 \\
\hline 质量/kg & $2.798 \times 10^{5}$ & $2.347 \times 10^{5}$ & -16.119 \\
\hline $\begin{array}{l}\text { 转动惯量 } \\
/\left(\mathrm{kg} \cdot \mathrm{m}^{2}\right)\end{array}$ & $1.070 \times 10^{9}$ & $8.591 \times 10^{8}$ & -19.710 \\
\hline
\end{tabular}

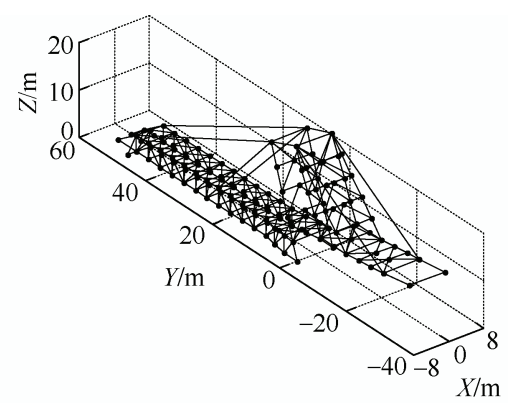

(a) 优化前斗轮取料机结构图

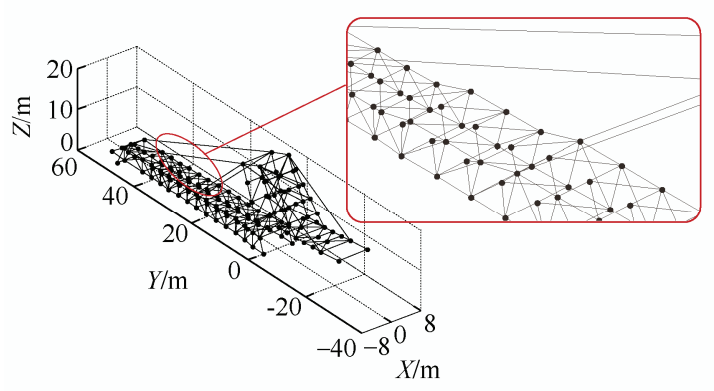

(b) 优化后斗轮取料机结构图

图 9 优化前与优化后的结构布局对比 
由图 $10 \mathrm{a}$ 可知, 斗轮取料机变形由优化前的 $150 \mathrm{~mm}$ 增大至 $162 \mathrm{~mm}$, 最大变形位置仍发生在斗 轮取料机悬臂的最前端, 最大变形幅值增大了 $8 \%$, 造成这个现象的主要原因是悬臂的长度在保持不 变的情况下, 悬臂的质量相对初始结构减小; 同时, 斗轮取料机平衡架的长度相对初始结构缩短了 $6.811 \mathrm{~m}$, 并且整个机构的高度降低后有效的改善 了悬臂受力分布。

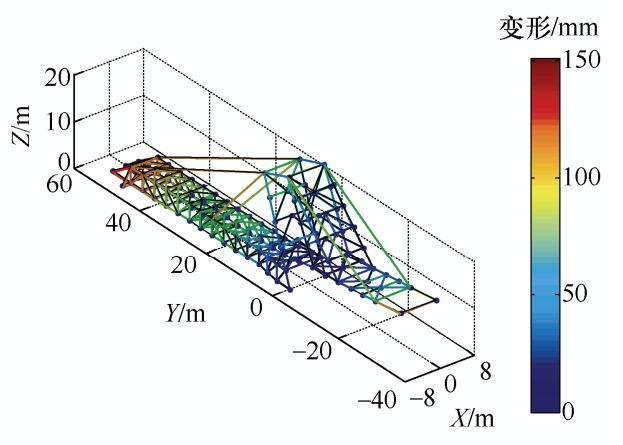

(a) 优化前取料机变形云图

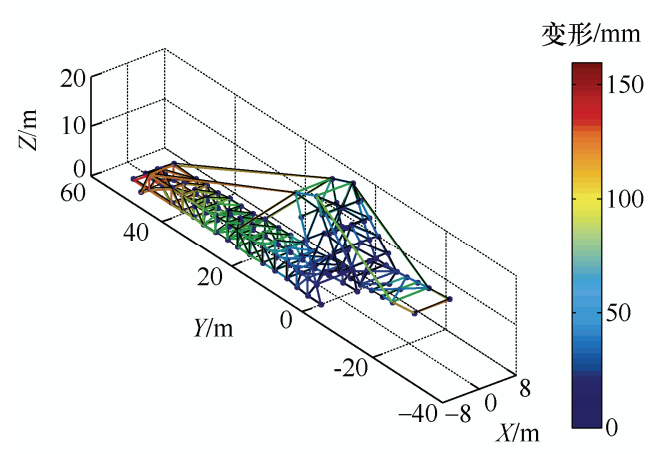

(b) 优化后取料机变形云图

图 10 优化前与优化后的变形对比

图 11 为优化前后结构的应力云图, 对比图中结 果可知, 优化前与优化后最大应力的位置均为悬臂 安装处附近, 主要原因是在优化模型中约束了所有 杆件最大应力。为了使优化后的结构仍能够满足异 常载荷工况要求, 在进行优化时, 每根杆件的最大 应力值应不允许大于初始结构的最大应力值, 优化 后的斗轮取料机结构尺寸更加紧凑, 组成斗轮取料 机系统的杆件参数也相对更为合理。

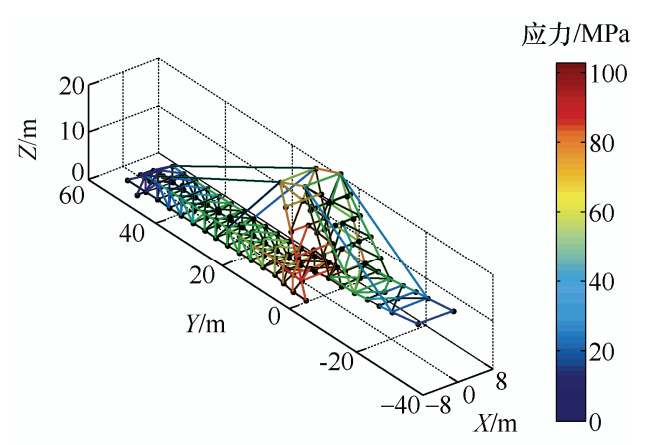

(a) 优化前结构的应力云图

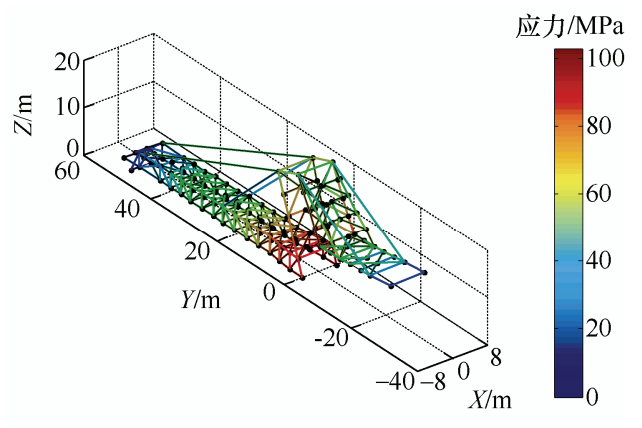

(b) 优化后结构的应力云图

图 11 优化前与优化后的结果对比

为获得优化后斗轮取料机的动力学响应, 基于 优化后的数据对取料机进行建模和分析。采用模态 参数和模态坐标对原微分方程进行表述, 从而使方 程更容易解耦, 求解后获得的每一列数据即为结构 系统的模态振型。通过对斗轮取料机进行计算, 得 到了斗轮取料机的振动频率和振型, 模态分析结果 如表 4 所示。为获得优化前和优化后的变化规律, 选取了 2 个典型振型进行分析, 如图 12 所示。

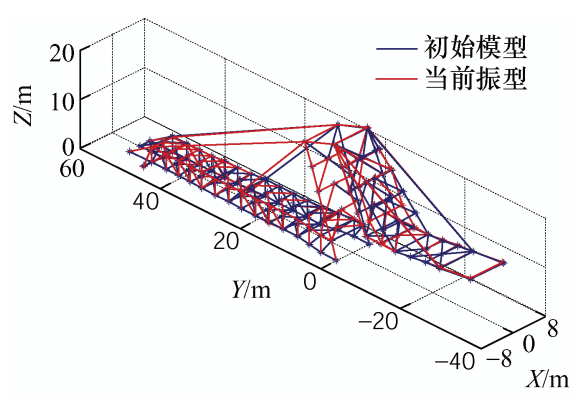

(a) 2 阶振型

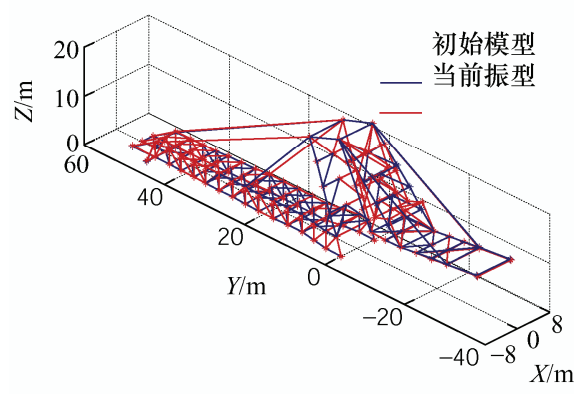

(b) 5 阶振型

图 12 斗轮取料机工作装置的振型

表 4 斗轮取料机模态分析结果

\begin{tabular}{ccc}
\hline 阶数 & 振型描述 & 频率 $/ \mathrm{Hz}$ \\
\hline 1 & 沿 $X$ 向振动 & 0.175 \\
2 & 沿 $Y$ 向伸缩振动 & 0.973 \\
3 & 沿 $Z$ 向扭动 & 1.131 \\
4 & 沿 $X$ 向弯扭振动 & 1.659 \\
5 & 沿 $Z$ 向上下振动 & 1.904 \\
6 & 沿 $Y$ 向扭动 & 2.813 \\
\hline
\end{tabular}

对比表 4 中的振型和频率值可知, 各阶振型的 
变化和频率值均不相同。根据现场测试和经验, 斗 轮取料机在取料过程中斗轮切削料堆的平均频率约 为 $0.77 \mathrm{~Hz}$, 经对比表 4 中工作装置的固有频率值, 结果表明优化后的结构布局方案是切实可行的。

\section{4 结论}

（1）针对传统优化算法在求解高维问题时存在 效率低、精度低的不足, 基于自然现象和物理现象, 提出将空气阻力和库仑力策略与传统的蜻蜓算法相 结合, 获得一种快速高精度的改进型蜻蜓算法, 并 通过测试函数验证了改进蜻蜓算法的收敛精度、稳 定性和计算时间。结果表明, 改进的蜻蜓算法适合 用于求解高维多目标优化问题。

（2）基于改进后的蜻蜓算法对斗轮取料机进行 质量和转动惯量多目标优化研究, 同时考虑系统的 可靠性和振动等强约束, 得到多目标优化 Pareto 前 沿解集。以其中一个最优解为例, 进行了静力学和 动力学特性计算, 结果表明斗轮取料机的结构改进 为截面阶梯结构布局更加合理, 主要性能指标均得 到大幅优化提升。

（3）本文的研究结果对蜻蜓算法等进化算法在 工程中的应用具有一定的参考价值，同时对国产取 料机的改进优化与创新设计也具有很好的参考价值 和指导意义。

\section{参 考 文 献}

[1] FILATOV G. Optimum designing of the bearing structures of bucket wheel excavators. three-mass model[J]. Journal of Emerging Technology and Advanced Engineering, 2017, 7(10): 378-383.

[2] DORN W, GOMORY R, GREENBERG H. Automatic design of optimal structures[J]. Design Mechaniuque, 1964, 3(1): 25-52.

[3] RUSIŃSKI E, MOCZKO P, KACZYŃSKI P. Structural modifications of excavator's bucket wheel by the use of numerical methods[C]//Solid State Phenomena. Trans Tech Publications, 2010, 165: 330-335.

[4] SHANG P, HU Y Z, HE L H, et al. The modal analysis of the main steel structure of bucket wheel stacker reclaimer[C]//Advanced Materials Research. Trans Tech Publications, 2013, 690: 3121-3124.

[5] 金华英.斗轮堆取料机前臂架结构优化与设计分析 [J]. 内燃机与配件, 2018(23): 205-206.

JIN Huaying. Structural optimization and design analysis of forearm of bucket wheel stacker reclaimer[J]. Internal Combustion Engine \& Parts, 2018, 690: 3121-2124.

[6] YUAN Y L, LÜ L Y, WANG S B, et al. Multidisciplinary co-design optimization of structural and control parameters for bucket wheel reclaimer[J]. Frontiers of Mechanical Engineering, 2020, 15(3): 406-416.

[7] YUAN Y L, LÜ L Y, WANG X B, et al. Optimization of a frame structure using the coulomb force search strategy-based dragonfly algorithm[J]. Engineering Optimization, 2020, 52(6): 915-931.

[8] YUAN Y L, SONG X G, SUN W , et al. Multidisciplinary design optimization of the belt drive system considering both structure and vibration characteristics based on improved genetic algorithm[J]. AIP Advances, 2018, 8 (5): 055115 .

[9] ZHAO M P. GA-based optimal design of derricking balance mechanism of bucket wheel stacker reclaimer[C]// Advanced Materials Research. Trans Tech Publications, 2012, 562: 672-675.

[10] 钱云芳, 蔡岗础. 大型斗轮堆取料机拉杆的受力分析与 优化 [J]. 港口装卸，2018(6): 6-10.

QIAN Yunfang, CAI Gangchu. Stress analysis and optimization of drawbar of large bucket wheel stacker reclaimer[J]. Port Operation, 2018(6): 6-10.

[11] 王九生, 王海斌. DQLK1000/1200.30 型斗轮堆取料机 回转机构改型优化 [J]. 酒钢科技, 2019(3): 53-56.

WANG Jiusheng, WANG Haibin. Modification and optimization of slewing mechanism for DQLK 1000/1200.30 bucket wheel stacker reclaim[J]. JISCO Technology, 2019(3): 53-56.

[12] 吴奋敬. 螺旋卸船机相对旋转式取料装置取料机理及 仿真研究[D]. 武汉: 武汉理工大学, 2017.

WU Feijing. Research of reclaiming mechanism and simulation of relatively-rotating inlet device of screw unloader[D]. Wuhan: Wuhan University of Technology, 2017.

[13] LIU S Y, LI J, HE H. Topological optimization of the main girder web based on $\mathrm{ESO}[\mathrm{J}]$. Journal of Engineering Design, 2011, 18(3): 174-177.

[14] SUN W, PENG X, DOU J, et al. Surrogate-based weight reduction optimization of forearm of bucket-wheel stacker reclaimer[J]. Structural and Multidisciplinary Optimization, 2020, 61(3): 1287-1301.

[15] 龚建民. 悬臂斗轮堆取料机总体设计及机构分析与优 化设计 $[\mathrm{J}]$. 水利水电施工，2016，157(4)：60-67. 
GONG Jianmin. Overall design and mechanism analysis and optimization design of cantilever bucket wheel stacker and reclaimer[J]. Water Conservancy and Hydropower Construction, 2016， 157(4): 60-67.

[16] 李军. 基于散体力学的堆取料机料斗结构优化设计 [D]. 天津: 河北工业大学, 2018.

LI Jun. Optimization design of hopper structure of heap reclaimer based on bulk mechanics[D]. Tianjin: Hebei University of Technology. 2018.

[17] WANG X B, SUN W, LI E Y, et al. Energy-minimum optimization of the intelligent excavating process for large cable shovel through trajectory planning [J]. Structural and Multidisciplinary Optimization, 2018, 58(5): 1-19.

[18] MIRJALILI S. Dragonfly algorithm: a new meta-heuristic optimization technique for solving single-objective, discrete, and multi-objective problems[J]. Neural Computing and Applications, 2016, 27(4): 1053-1073.

[19] YANG X S. Nature-inspired metaheuristic algorithms[M]. Beckington: Luniver Press, 2008.

[20] PAVLYUKEVICH I. Levy flights, non-local search and simulated annealing[J]. Mathematics, 2007, 226(2): 1830-1844.

[21] 徐遥, 王士同. 引力搜索算法的改进 [J]. 计算机工程与 应用, 2011，47(35): 188-192.

XU Yao , WANG Shitong. Enhanced version of graritaflonal search algorithm : weighted GSA[J]. Computer Engineering and Applications. 2011，47(35): 188-192.

[22] 刘俊波, 王可人, 冯辉, 等. 一种基于认知引擎的 $\mathrm{t}$ 分 布变异萤火虫算法 [J]. 数据采集与处理, 2015(4): 909-914.

LIU Juntong, Wang Keren, FENG Hui, et al. GSO algorithm with $\mathrm{t}$-distribution mutation for cognitive engine[J]. Journal of Data Acquisition and Processing,
2015 (4): 909-914.

[23] MIRJALILI S. Moth-flame optimization algorithm: A novel nature-inspired heuristic paradigm[J]. Knowledge-Based Systems, 2015, 89: 228-249.

[24] LI Z , ZHOU Y , ZHANG S , et al. Lévy-flight moth-flame algorithm for function optimization and engineering design problems[J]. Mathematical Problems in Engineering, 2016, 2016(8): 1-22.

[25] IRUTHAYARAJAN M W, BASKAR S. Covariance matrix adaptation evolution strategy based design of centralized PID controller[J]. Expert Systems with Applications, 2010, 37(8): 5775-5781.

[26] SONG J M, LI S P. Elite opposition learning and exponential function steps-based dragonfly algorithm for global optimization[C]//2017 IEEE International Conference on Information and Automation (ICIA). IEEE, 2017, 1178-1183.

[27] 徐建国. 轴流㬌叶片应力与模态分析[D]. 扬州: 扬州大 学, 2008 .

XU Jianguo. Stress and modal analysis of axial-flow pump blades[D]. Yangzhou: Yangzhou University, 2008.

[28] COELLO, C A. An updated survey of GA-based multiobjective optimization techniques[J]. Acm Computing Surveys, 2000, 32(2): 109-143.

[29] YAPO P O , GUPTA H V, SOROOSHIAN S. Multi-objective global optimization for hydrologic models[J]. Journal of Hydrology, 1998, 204(1-4): 83-97.

作者简介: 宋学官(通信作者), 男, 1982 年出生, 博士, 教授, 博士研 究生导师。主要研究方向为多学科耦合建模与协同优化、代理模型技术、 工业大数据挖掘、数字孪生与装备智能化等。

E-mail:sxg@dlut.edu.cn

原永亮, 男, 1989 年出生, 博士, 讲师。主要研究方向为多学科协同优 化、群智能优化算法、基于仿真的优化设计。

E-mail: yuanyongliang@hpu.edu.cn 